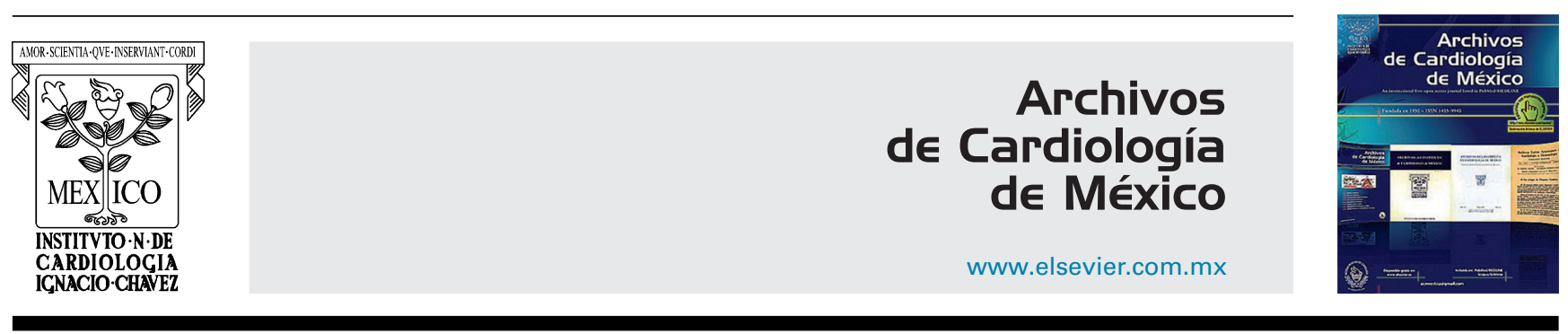

EDITORIAL

\title{
Afección cardíaca de la enfermedad de Lyme: ¿Por qué México debe de prestar atención a este tema?
}

\section{Lyme carditis: Why should Mexico pay attention to this problem?}

\author{
Diego R. Sánchez-Vázquez ${ }^{\mathrm{a}}$, Manlio F. Márquez ${ }^{\mathrm{b}}$ y Adrian Baranchuk ${ }^{\mathrm{c}, *}$ \\ a Departamento de Competitividad y Salud, Fundación Mexicana para la Salud, Ciudad de México, México \\ b Departamento de Electrocardiografía, Instituto Nacional de Cardiología Ignacio Chávez, Ciudad de México, México \\ c Arrhythmia Service, Queen's University, Kingston, Ontario, Canadá
}

Recibido el 27 de marzo de 2018; aceptado el 23 de abril de 2018

\section{Introducción}

En un mundo globalizado, con un aumento significativo en la migración y desplazamiento de personas, como se observa en la frontera entre la República Mexicana y los Estados Unidos de América (EE. UU.), existe no solo un intercambio constante de bienes y productos, sino también de patógenos transmisibles entre un país y otro ${ }^{1,2}$. Por otra parte, el aumento en la densidad poblacional en esta zona transfronteriza acarrea que nuevas poblaciones de seres humanos se establezcan en distintas áreas geográficas ${ }^{1}$. De esta manera, se modifica el ecosistema natural donde se establecen, aumentando la posibilidad de contacto con enfermedades transmisibles por vectores $\mathrm{y}$, por ende, la posibilidad del deterioro de la salud en estas poblaciones ${ }^{1}$. Un ejemplo claro de este tipo de enfermedad transmisible por vectores es la borreliosis o enfermedad de Lyme, ocasionada por

\footnotetext{
* Autor para correspondencia. 76 Stuart St, Kingston, ON K7L 3N6, Canadá. Teléfono: +1 613549 6666, ext. 3801

Correo electrónico: Adrian.Baranchuk@kingstonhsc.ca (A. Baranchuk).
}

picadura de garrapatas Ixodes scapularis (I. scapularis) e Ixodes pacificus, que son las que predominan en el continente norteamericano ${ }^{2,3}$. Esta infección es transmitida al humano por picadura de garrapatas infectadas por la espiroqueta Borrelia burgdorferi (B. burgdorferi), que se alojan en distintos reservorios animales ${ }^{2}$. El venado de cola blanca (Odocoileus virginianus) es portador de la garrapata en su forma adulta, favoreciendo la infección de otros mamíferos más pequeños como los ratones de patas blancas (Peromyscus leucopus) ${ }^{3}$.

\section{Afección cardiaca en la enfermedad de Lyme}

Las manifestaciones de la enfermedad de Lyme se dividen en 3 estadios:

- Infección temprana localizada: posterior a la picadura de la garrapata existe un periodo de incubación de 3 a 32 días que precede a la lesión clásica, conocida como eritema migrans ${ }^{3}$.

- Infección temprana diseminada: su inicio puede variar desde la primera semana posterior a la infección hasta 
meses más tarde ${ }^{3}$. En este estadio existe una diseminación de espiroquetas al sistema nervioso, músculo esquelético y corazón ${ }^{4}$. El corazón se ve afectado en un $5 \%$ de los $\operatorname{casos}^{5}$. La invasión producida directamente en los distintos niveles del tejido cardiaco por las espiroquetas de $B$. burgdorferi es conocida como carditis de Lyme $^{6}$. En esta fase evolutiva, ocurre la mayoría de las manifestaciones cardiacas de la enfermedad de Lyme ${ }^{4}$.

- Infección tardía: es cuando la enfermedad persiste entre uno a 3 años en el organismo y puede resultar en miocardiopatía dilatada y encefalopatía ${ }^{3,4}$. Puede también producirse una disautonomía difícil de diagnosticar, ya que los síntomas son inespecíficos y comunes a otras enfermedades (p. ej., fatiga, mareos, cefalea, irritabilidad, insomnio $)^{3}$. Estos pacientes, a pesar de haber recibido tratamiento adecuado, persisten con estas manifestaciones clínicas, asociándose a la gravedad de la enfermedad al inicio y al retraso en el tratamiento ${ }^{3}$.

En la carditis de Lyme se produce una infiltración transmural de linfocitos, células plasmáticas y macrófagos en las 3 capas del corazón: endocardio, miocardio y pericardio ${ }^{4,7}$. A pesar de ser una manifestación poco frecuente, puede producir síntomas graves, como insuficiencia cardiaca y blo- queo aurículo-ventricular completo ${ }^{4,7}$. Estas complicaciones son más frecuentes en hombres (3:1) y más graves en pacientes sintomáticos que no reciben tratamiento temprano ${ }^{4,7,8}$. Después de la manifestación cutánea (eritema migrans), las alteraciones cardiacas pueden aparecer en las siguientes semanas ${ }^{4,7}$. Es importante señalar que muchos pacientes ni siquiera recuerdan haber sido picados por garrapatas ni haber tenido alguna manifestación cutánea ${ }^{6}$. Los síntomas cardiovasculares más frecuentes son: síncope (40\%), disnea $(33 \%)$, sensación de aturdimiento (33\%), palpitaciones (22\%) y dolor torácico $(20 \%)^{4,6,7}$. La exploración física generalmente es normal, pero debe sospecharse el diagnóstico si se detecta bradicardia y confirmarse con un electrocardiograma de 12 derivaciones ${ }^{4,6}$. Algunos refieren síntomas concomitantes de manifestaciones sistémicas asociadas, como dolor en articulaciones o síntomas neurológicos ${ }^{4}$. Aunque las alteraciones en el sistema de conducción sean más frecuentes, también han sido descritos casos con fibrilación auricular, bloqueos de rama y prolongación del intervalo $\mathrm{QT}^{6-8}$.

Dentro de las pruebas serológicas diagnósticas se incluye la de ELISA, para detección de anticuerpos $\lg M$ e $\operatorname{lgG}^{4}$. No obstante, un resultado negativo no descarta la enfermedad ${ }^{4}$. Los resultados obtenidos por serología deben de ser interpre-
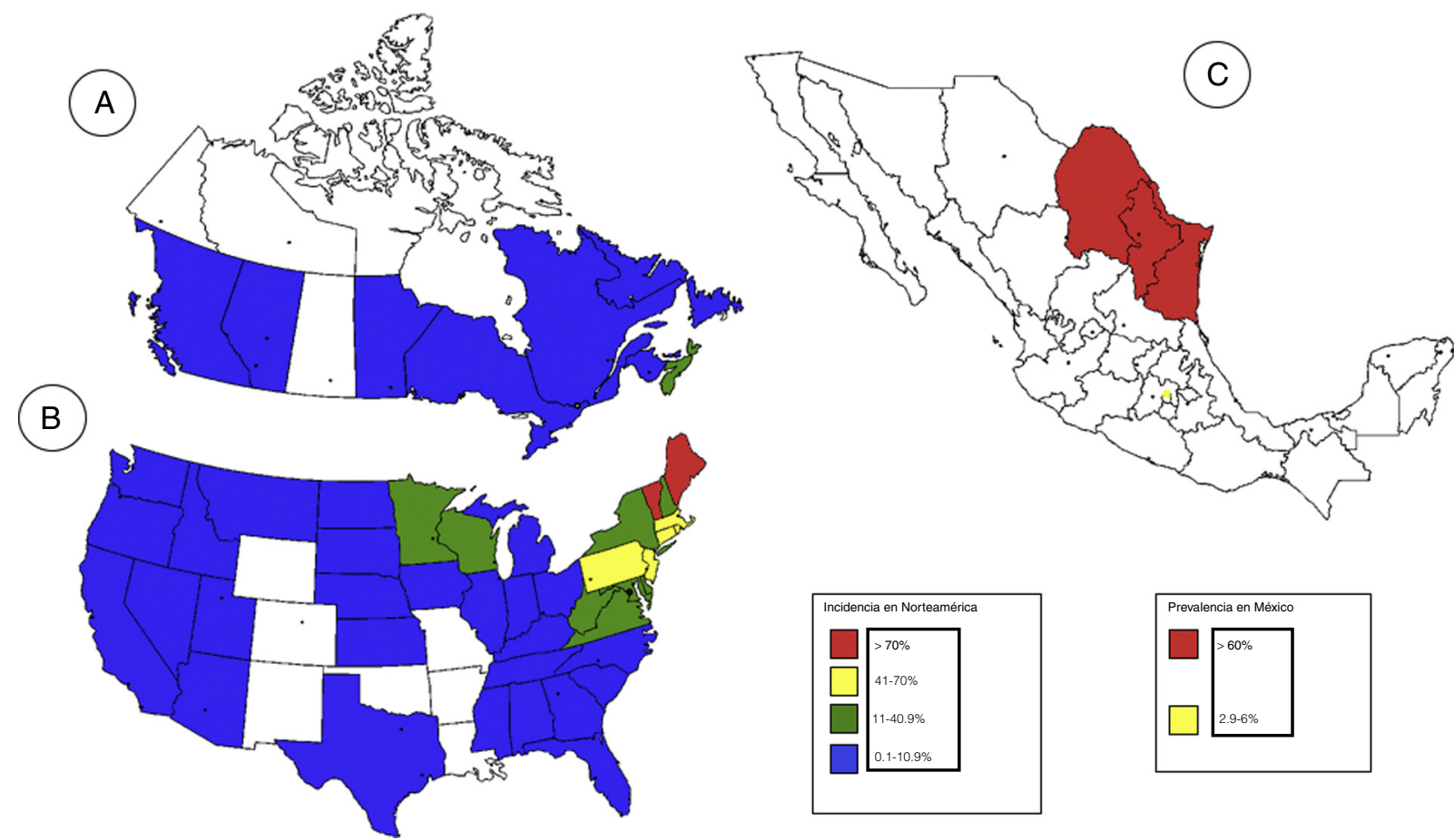

Figura 1 Incidencia de la enfermedad de Lyme en EE. UU. y Canadá durante el año 2015, y prevalencia de la enfermedad de Lyme en México durante el año 2003.

Panel A: Mapa perteneciente a Canadá. Nótese que la provincia con mayor incidencia durante ese año fue Nova Scotia (26.1\%). Toda la frontera con EE. UU., excepto Saskatchewan se considera endémica para enfermedad de Lyme. Reproducido con modificaciones de la pagina web oficial del gobierno de Canadá ${ }^{10}$.

Panel B: Mapa perteneciente a EE.UU. El 95\% de los casos nuevos de enfermedad de Lyme durante 2015 sucedieron en la frontera noreste con Canadá. Se observa que es una enfermedad emergente en la frontera sureste con México. Reproducido con modficaciones de la pagina web oficial de la CDC (Centers for Disease Control and Prevention) ${ }^{9}$.

Panel C: Mapa perteneciente a México. El último estudio donde se demuestra la prevalencia de la enfermedad de Lyme en México fue en 2003. Desde entonces, México no cuenta con un análisis que demuestre su prevalencia o incidencia actual de este padecimiento. La mayor prevalencia durante ese año se presenta en el noreste del país, siendo la frontera noreste entre México y EE. UU. Reproducido con modificaciones del estudio epidemiológico de Gordillo- Pérez et al., ${ }^{11}$. 
tados teniendo en cuenta el contexto clínico del paciente, puesto que actualmente existe una falta de estandarización en los métodos de diagnóstico, presentando una especificidad y sensibilidad limitada ${ }^{4}$.

\section{Epidemiología en Norteamérica}

La enfermedad de Lyme es muy común en Norteamérica ${ }^{7}$. En EE. UU., durante 2015 el 95\% de los casos confirmados de enfermedad de Lyme fueron reportados en el noreste del país, predominantemente en estados como: Vermont (incidencia del 78.4\%), Maine (74.7\%), Pennsylvania (57.4\%), Rhode Island (53.4\%), Connecticut (52.2\%), New Jersey (43.9\%), entre otros estados (fig. 1) $)^{9}$. Así mismo, estudios actuales indican que el sureste de EE. UU. podría también ser considerado como zona endémica ${ }^{2}$. Esta zona colinda con México, donde se ha demostrado que I. scapularis es considerado como el vector principal de B. burgdorferi ${ }^{2}$.

Por otra parte, en Canadá existe una importante incidencia de esta enfermedad, fundamentalmente en la zona limítrofe con EE. UU. ${ }^{10}$. Durante el año 2016, el gobierno de Canadá informó 987 casos confirmados de enfermedad de Lyme, esto es 26 veces menos de lo reportado en EE. UU. en el mismo año ${ }^{9,10}$. No obstante, la enfermedad de Lyme es actualmente considerada como endémica en Canadá, principalmente en las provincias de: Nova Scotia (incidencia del 26.1\%), Ontario (3.1\%), Prince Edward Island (2.7\%), Manitoba $(2.4 \%)$ y Quebec (1.9\%) (fig. 1$)^{10}$.

\section{Epidemiología en México}

Entre EE. UU. y México existe una frontera de 3,100 km, donde se produce un intercambio de personas de manera constante $^{1}$. Aunque algunos estudios indican que esta enfermedad puede ser endémica en México (principalmente, en el norte del país), actualmente se desconoce su epidemiología ${ }^{2,11}$. Con las estadísticas disponibles en este momento, podemos formular la siguiente pregunta: ¿qué regiones en México son las más prevalentes en enfermedad de Lyme?

La presencia de garrapatas Ixodes infectadas en el norte del país indican que esta zona pudiera ser considerada endémica para enfermedad de Lyme; así mismo, esta puede representar un riesgo de transmisión de $B$. burgdorferi al humano ${ }^{3}$. En una revisión reciente, en la que tratan de buscar una relación entre las poblaciones adyacentes al Río Grande y la prevalencia de B. burgdorferi a lo largo del mismo, demostró que los estados de Tamaulipas, Nuevo León y Coahuila presentan la mayor seroprevalencia del país, siendo el reservorio más frecuente el venado de cola blanca. Esta revisión hace referencia al estudio epidemiológico de Gordillo-Pérez et al. ${ }^{11}$, en el que demostraron que la seroprevalencia para la enfermedad de Lyme en el noreste de la República Mexicana (2, 346 pacientes) fue del 6.2\% (fig. 1$)^{1}$. De esta manera, existe una alta prevalencia de $B$. burgdorferi en la región noreste de México. Por lo tanto, la frontera entre Texas y México aparentemente es un paso transfronterizo constante en la patogenicidad de la enfermedad de Lyme. No obstante, el norte de México no es la única región donde se ha reportado prevalencia de

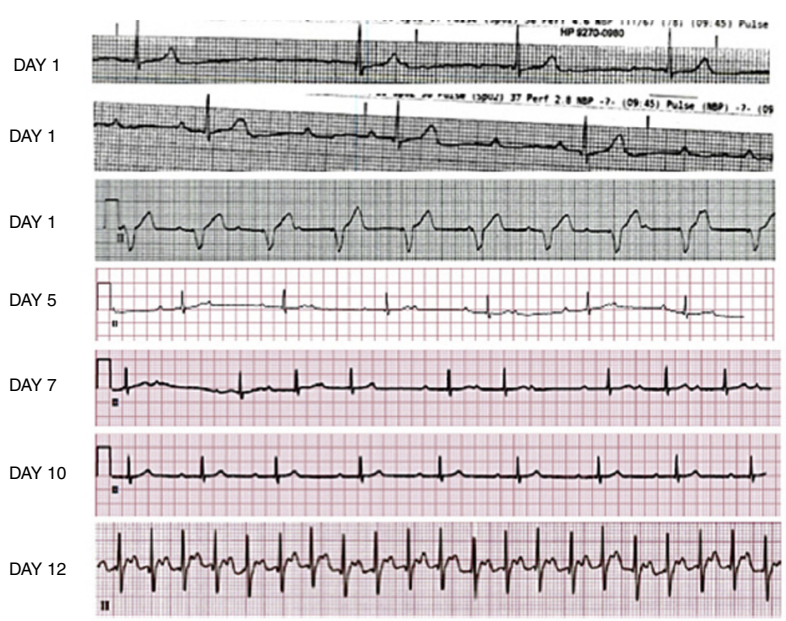

Figura 2 Evolución electrocardiográfica de un paciente con carditis de Lyme.

La carditis de Lyme se caracteriza por una evolución rápida de sus alteraciones en el sistema de conducción. Progresando rápidamente de un bloqueo AV de primer grado a uno de tercer grado ${ }^{6}$. En la siguiente figura se observa una rapida progresion a bloqueo AV de alto grado e implante de marcapasos transitorio (DAY 1) y una progresiva mejoria del trastorno de conduccion en los dias subsiguientes, hasta normalizarse la conduccion. Antes del alta, una prueba de esfuerzo demuestra conduccion 1:1 a una frecuencia de 175 latidos por minutoe (DAY 12).

B. burgdorferi. Existen estudios en los cuales se refieren casos de enfermedad de Lyme en el centro y sur del país, incluyendo: la Ciudad de México (centro del país) y Yucatán (sureste del país) ${ }^{11,12}$. Cabe resaltar que donde se reportaron estos contagios, se controló el hecho que los pacientes no tuvieren el antecedente de haber salido o viajado fuera de las zonas de contagio para evitar sesgar el resultado ${ }^{12}$.

\section{Manejo del bloqueo AV de alto grado}

Estudios de caso y series de casos han propuesto que el bloqueo aurículo-ventricular de tercer grado es el más frecuentemente involucrado en la carditis de Lyme $^{7,8}$. La presentación más habitual se da en hombres de entre 15-45 $a_{n ̃ o s} 6,7$. Es muy frecuente que un paciente evolucione de un bloqueo de primer grado a uno de tercer grado en cuestión de días, horas, incluso minutos (fig. 2$)^{4,7}$. Debido a esto, se indica que a todo paciente con sospecha de carditis de Lyme se hospitalice para monitorización electrocardiográfica y que se comience con el tratamiento antibiótico de manera precoz ${ }^{8}$. Puesto que el diagnóstico y el tratamiento antibiótico temprano pueden curar y revertir el bloqueo AV de cualquier grado, la sospecha debe inducir al tratamiento precoz por vía intravenosa ${ }^{8}$. La duración total del tratamiento antibiótico es de entre $2-4$ semanas ${ }^{8}$. El bloqueo $\mathrm{AV}$ revierte entre 7 a 10 días, siendo que algunos pacientes requerirán marcapasos transitorio ${ }^{4,8}$. Por conseguiente, ante un hombre de entre 15 a 45 años, con un bloqueo AV de alto grado sin causa aparente, se debe de pensar en carditis de Lyme antes de considerar un marcapasos permanente ${ }^{8}$. De esta manera, se evitarán implantes innecesarios, ya que la mayoría de los bloqueos se resolverán solo con tratamiento 
antibiótico $^{8}$. Esta estrategia beneficia no solo al paciente joven, sino al sistema de salud a evitar gastos innecesarios.

\section{Conclusiones}

En México, la enfermedad de Lyme puede ser considerada como endémica, principalmente en el noreste del país. Con este editorial deseamos enviar un mensaje a la comunidad médica de México para el reconocimiento temprano de la carditis de Lyme. Se debe sospechar en pacientes jóvenes con bloqueo AV de alto grado y sin causa aparente del mismo. El diagnóstico y el tratamiento precoz reducen la necesidad de implante de marcapasoss de estos pacientes.

\section{Financiación}

La presente investigación no ha recibido ayudas específicas provenientes de agencias del sector público, sector comercial o entidades sin ánimo de lucro.

\section{Conflicto de intereses}

Los autores declaran no tener ningún conflicto de intereses.

\section{Bibliografía}

1. Esteve-Gassent MD, Pérez de León AA, Romero-Salas D, FeriaArroyo TP, Patino R, Castro-Arellano I, et al. Pathogenic Landscape of Transboundary Zoonotic Diseases in the MexicoUS Border Along the Rio Grande. Front Public Heal. 2014;2: $1-23$.
2. Feria-Arroyo TP, Castro-Arellano I, Gordillo-Perez G, Cavazos AL, Vargas-Sandoval M, Grover A, et al. Implications of climate change on the distribution of the tick vector Ixodes scapularis and risk for Lyme disease in the TexasMexico transboundary region. Parasites and Vectors. 2014;7: $1-16$.

3. Portillo A, Santibáñez S, Oteo JA. Enfermedad de Lyme. Enferm Infecc Microbiol Clin. 2014;32 SUPPL.1:37-42.

4. Scheffold N, Herkommer B, Kandolf R, May AE. Lyme Carditis - Diagnosis, Treatment and Prognosis. Dtsch Arztebl Int. 2015;112:202-9.

5. Earl TJ. Cardiac manifestations of Lyme disease. Med Health R I. 2010;93:339-41.

6. Fuster LS, Gul EE, Baranchuk A. Electrocardiographic progression of acute Lyme disease. Am J Emerg Med. 2017;35:1040e5-6.

7. Robinson ML, Kobayashi T, Higgins Y, Calkins H, Melia MT. Lyme Carditis. Infect Dis Clin North Am. 2015;29:255-68.

8. Wan D, Blakely C, Branscombe P, Suarez-Fuster L, Glover B, Baranchuk A. Lyme Carditis and High-Degree Atrioventricular Block. Am J Cardiol. 2018.

9. Centers for Disease Control and Prevention. Lyme Disease [Internet]. 2017. Consultado el 15 de marzo de 2018. https://www.cdc.gov/lyme/stats/index.html.

10. Government of Canada. Surveillance of Lyme disease [Internet]. 2017. Consultado el 14 de marzo de 2014. https://www.canada.ca/en/public-health/services/diseases/ lyme-disease/surveillance-lyme-disease.html.

11. Gordillo-Pérez G, Torres J, Solórzano-Santos F, GarduñoBautista V, Tapia-Conyer R, Muñoz O. Estudio seroepidemiológico de borreliosis de Lyme en la Ciudad de México y el noreste de la República Mexicana. Salud Publica Mex. 2003;45: $351-5$.

12. Gordillo-Pérez G, Torres J, Solórzano-Santos F, De Martino $\mathrm{S}$, Lipsker D, Velázquez E, et al. Borrelia burgdorferi infection and cutaneous lyme disease. Mexico. Emerg Infect Dis. 2007;13:1556-8. 